\title{
Philosophiques
}

\section{Hannah Arendt, Juger. Sur la philosophie politique de Kant, (suivi de deux essais interprétatifs par Ronald Beiner et Myriam Revault d'Allonnes), Paris, Seuil, 1991, 244 pages.}

\section{Lukas K. Sosoe}

Volume 22, numéro 1, printemps 1995

URI : https://id.erudit.org/iderudit/027323ar

DOI : https://doi.org/10.7202/027323ar

Aller au sommaire du numéro

Éditeur(s)

Société de philosophie du Québec

ISSN

0316-2923 (imprimé)

1492-1391 (numérique)

Découvrir la revue

Citer ce compte rendu

Sosoe, L. K. (1995). Compte rendu de [Hannah Arendt, Juger. Sur la philosophie politique de Kant, (suivi de deux essais interprétatifs par Ronald Beiner et Myriam Revault d'Allonnes), Paris, Seuil, 1991, 244 pages.] Philosophiques, 22(1), 187-190. https://doi.org/10.7202/027323ar d'utilisation que vous pouvez consulter en ligne.

https://apropos.erudit.org/fr/usagers/politique-dutilisation/ 
Hannah Arendt, Juger. Sur la philosophie politique de Kant, (suivi de deux essais interprétatifs par Ronald Beiner et Myriam Revault d'Allonnes), Paris, Seuil, I99I, 244 pages.

\section{par Lukas K. Sosoe}

Le présent ouvrage est la première traduction française du livre de Hannah Arendt paru, en anglais, sous le titre Lectures on Kant's Political Philosophy (Chicago University Press, 1982). Le livre, on le sait, représente la troisième partie de La vie de lesprit. Il était censé, avec La Pensée et Vouloir, constituer une trilogie. Tout porte à croire que Hannah Arendt l'a laissé inachevé. D'où la difficulté qu'il y a à lui donner une interprétation à la fois précise et cohérente. Tout comme la version anglaise, la traduction française est suivie d'un Essai interprétatif (129-216) et se complète par un autre essai, interprêtatif lui aussi, écrit par la traductrice de l'ouvrage : Myriam Revault d'Allonnes (217-239), à laquelle nous devons une traduction réussie. Une réussite qui nous décharge de nous attarder sur les mérites de cette édition qui, tout en parlant pour ellemême et ses qualités, offre au public francophone l'accès à cet ouvrage dont nous aimerions plutôt souligner l'actualité. Celle-ci ne se perçoit dans son entier qu'à travers les thèmes centraux que Hannah Arendt y a développés et qui résultent, il faut à tout prix le souligner, d'une étonnante intuition interprétative.

Quelle que soit la lecture qu'on puisse en faire, Juger de Hannah Arendt est un livre quelque peu énigmatique. Ce n'est pas tant la forme qui, comme nous 
le soulignions, est restée à l'ébauche ou en forme de notes de cours que les éditeurs ont tenté de reconstituer, que la question philosophique qu'il pose : reconstruire une catégorie importante de l'esthétique kantienne, plus précisément de la Critique de la faculté de juger en soulignant sa fécondité pour une philosophie de l'espace public. Fécondité pour une philosophie politique qui fait dire à Arendt qu'il existe, chez Kant, deux philosophies politiques, celle des écrits politiques proprement dits de Kant et celle que développe, bien entendu indirectement, la troisième Critique. Aussi écrit-elle que Kant:

Expose deux philosophies politiques, sensiblement différentes l'une de l'autre : la première est celle qui est communément reçue comme telle dans la Critique de la raison pratique et l'autre est contenue dans la Critique de la faculté de juger (CFJ). Que la première partie de la Critique de la faculté de juger. constitue, en réalité, une philosophie politique est un fait que les travaux sur Kant mentionnent rarement; en revanche, il ressort, selon moi, de tous ses écrits politiques que Kant accorde au thème du "jugement" beaucoup plus de poids qu'à celui de la "raison pratique" (Freedom and Politics, p. 207).

Étonnante thèse qu'Arendt défendra pourtant. Pour Kant, le jugement crée, entre les hommes, la communication. En effet, en jugeant du beau, j'élève une prétention à la validité pour tous, sans universalité fondée objectivement (CFJ § 6). Cette prétention, souligne Kant, est paradoxalement une prétention subjective à l'universalité, dans la mesure où ce qui est beau l'est pour moi. Encore faut-il, pour que cette prétention puisse être prise en compte par les autres et que je puisse en attendre une adhésion de leur part, que mon jugement soit communicable. Les autres doivent pouvoir comprendre mon jugement et l'apprécier. Toutefois les autres ne représentent pas ici les spécialistes, mais la communauté de tous ceux qui peuvent comprendre mon jugement et prendre position. Or, à l'exemple de toutes les connaissances, nos jugements doivent être communicables. Ceci présuppose, en faveur des autres, la capacité de comprendre. Seulement cette capacité doit être universelle, dans la mesure où tous doivent pouvoir comprendre mon jugement pour qu'un accord ou même un désaccord soit possible. Kant est explicite sur ce point : « comme cet accord lui-même doit pouvoir se communiquer universellement, et donc aussi par conséquent le sentiment de cet accord l...l, et comme la communicabilité universelle d'un sentiment présuppose à son tour un sentiment commun, c'est donc avec raison qu'on pourra admettre l'existence de ce dernier $\gg$. Nous avons là réunis les ingrédients qui ont permis à Hannah Arendt de transposer le jugement de goût sur un plan politique et de développer, à partir de celui-ci, une théorie de l'espace public qui, bien entendu, ne s'est pas contentée d'une répétition de Kant.

L'espace public, pour Hannah Arendt, est nécessairement un espace de communication sans laquelle le monde n'a pas de sens, tout comme sans l'homme, il n'aurait pas de sens. C'est en cela que le jugement de goût est commun à tout être humain " et nous révèle la nature du monde » qui est 
essentiellement politique (68). L'auteur va plus loin : la pensée critique implique communicabilité, donc faculté de juger dont l'exercice nous accorde un mode de pensée élargi. Nous nous mettons à la place des autres et les autres à notre place. Chaque prétention se trouve alors soumise à l'examen d'où résultera la compréhension. Même la réflexion solitaire présuppose l'existence des autres sans lesquels aucune pensée ne sera possible. Pour Arendt. Kant n'a pas rendu au concept de sens commun toute sa portée politique, dans le sens tout à fait aristotélicien du terme.

Espace public et réflexion, deux concepts déterminants, qui donneront à la théorie arendtienne du jugement deux différentes orientations : une orientation contemplative et une orientation politique. Toutes deux se fondent sur Kant. Alors que la première reprend l'idée de Kant développée dans le Conflit des Facultés au sujet de la Révolution française sur le sentiment d'enthousiasme dont est saisi l'observateur de la Révolution, la seconde se veut plus active. Ce n'est plus le jugement de celui qui se met à distance de l'événement et contemple son cours ainsi que les acteurs qui y sont impliqués, mais celui du citoyen qui participe à l'espace public et qui, par ses jugements, donne sens à la vie politique. donc humaine. C'est cette dernière conception politique, active, du jugement, qui dominera la pensée d'Hannah Arendt au point de la rapprocher d'une position quasi aristotélicienne. La communication entre les hommes devient alors essentiellement une communication politique. Comme le remarque à juste titre Beiner, « Arendt avait introduit la notion de jugement pour fonder plus solidement sa conception de l'action politique entendue comme l'action concertee d'une pluralité d'acteurs dans un espace public. Les êtres humains peuvent agir en tant qu'êtres politiques parce qu'ils sont susceptibles d'entrer dans des points de vue potentiels des autres; ils partagent le monde avec d'autres en jugeant ce qu'ils ont en commun et l'objet de leurs jugements en qualité d'êtres politiques, ce sont les paroles et les actes qui illuminent l'espace d'apparence » (I34).

On comprend dès lors pourquoi nous devons supposer le sens commun à tout le monde. Si personne ne doit être exclu de l'espace public, c'est que tous nous disposons d'une faculté de juger, une faculté qui ne s'apprend pas, mais comme dit Kant, s'exerce. On comprend également pourquoi Arendt privilègie la prudence et trouve, à partir de cette conception du jugement, son inspiration critique des effets destructeurs de la modernité, des sociétés contemporaines notamment, qui à travers leurs structures bureaucratiques, technocratiques et leur haute spécialisation, empêchent les hommes de discerner et les conduisent à la dépolitisation. Toutefois Arendt ne s'installe pas dans le pessimisme total, dans la critique pure et simple des sociétés contemporaines au profit des traditions et des valeurs qu'elles ont rendues caduques. L'appel au jugement nous est lancé pour recréer un espace public qui, pour nous inspirer encore une fois de Beiner, se fonde désormais sur « l'accord de nos jugements » 
au sein d'une communauté jugeante idéale, car le danger est de s'abstenir de juger - la banalité du mal -, de rester passif et de laisser la volonté capituler devant les forces du mal plutôt que d'exercer un jugement autonome ( $16 \mathrm{I})$.

Hier c'était des régimes totalitaires qu'Hannah Arendt parlait et qu'elle concevait comme une des terribles conséquences de l'avènement de la modernité. Aujourd'hui la menace semble être la dégradation complète des conditions d'une pensée pratique autonome qui, inexorablement, confie de plus en plus à des technocrates et à d'autres spécialistes - qu'ils s'appellent bioéthiciens ou éthiciens tout court, cela ne change rien - l'exigence de l'effort d'accorder nos jugements. Le jugement pratique qui est le propre de tout homme se trouve ainsi, à l'ère de l'éthique et du droit, pris en charge par des personnes spécialisées. Aussi les chances de créer un espace public, tel qu'Hannah Arendt le concevait. s'érodent-elles lentement, imperceptiblement. Il semble que lire aujourd'hui Hannah Arendt, et à travers elle, redécouvrir l'idéal d'une pensée qui redonne à l'être sa compétence critique, soit devenu aujourd'hui le passage obligé d'un véritable programme éthique et politique dans le sens large du terme, bref de la raison pratique reconstruite. Raison pratique qui, si l'on accepte la thèse arendtienne des deux philosophies politiques et les critiques du sujet pratique kantien, reste pure pratique, close sur elle-même et ne laisse pour toute possibilité de penser l'action que le jugement et la discussion.

Département de philosophie

Université de Montréal 